$\begin{gathered}\text { Revista do Departamento de Geografia } \\ \text { Universidade de São Paulo } \\ \text { www.revistas.usp.br/rdg }\end{gathered}$
Volume Especial - XVII SBGFA / I CNGF (2017)

\title{
Análise Estatística Anual e Mensal das Ondas Atuantes na Costa de Ilhéus (Bahia) Baseada na Reanálise de Série Temporal de 31 Anos do Modelo de Ondas WAVEWATCH III
}

\section{Annual and Monthly Statistical Analysis of Ocean Waves Offshore Ilhéus (Bahia) Based on a Thirty-One Year Time Series WAVEWATCH III Model Reanalysis}

\author{
Matheus Santos Lobo \\ Universidade Estadual de Santa Cruz \\ mlobo636@gmail.com
}

Bárbara Savina Silva Santos

Universidade Estadual de Santa Cruz bsavina94@gmail.com

Ana Amelia de Oliveira Lavenère-Wanderley Universidade Estadual de Santa Cruz amelia@uesc.br

\begin{abstract}
Resumo: Mobilidade e transporte de sedimentos induzidos por ondas são questões importantes em estudos do balanço sedimentar na zona costeira. A falta de dados disponíveis acerca do clima de ondas na costa brasileira dificulta e prejudica o entendimento da dinâmica costeira para o estabelecimento correto das ocupações na zona costeira. Uma série temporal de 31 anos (1979-2009) de parâmetros de ondas (altura significativa, período de pico e direção), obtidos do modelo WAVEWATCH III (NCEP NOAA), foi analisada estatisticamente, objetivando determinar a tendência central e a variabilidade das informações. O programa $\mathrm{R}$ para tabular e avaliar as informações, realizar os cálculos estatísticos e sua exibição gráfica. A análise anual da série temporal indicou a predominância de ondas provenientes de Leste e Sudeste, com alturas variando entre $0.39 \mathrm{~m}$ e $4.89 \mathrm{~m}$. Os períodos médios indicaram a predominância de ondas geradas por vento local (vagas), com valores inferiores a 10 segundos. A influência de ondas geradas em outras regiões do oceano (marulhos) é evidenciada quando considerado o desvio padrão do registro. Os parâmetros tabulados mensalmente indicaram como os mais energéticos os meses de junho, julho, agosto e setembro, que correspondem às estações inverno e primavera.
\end{abstract}

Palavras-chave: Parâmetros da Onda; Força da Onda; Programa R.

\begin{abstract}
Wave-induced sediment transport and mobility are important issues in sediment balance studies in the coastal zone. The lack of available data about the wave climate on the Brazilian coast makes it difficult to understand the coastal dynamics for the correct establishment of infrastructures in the coastal zone. A thirty-one year time series (1979-2009) of wave parameters (significant height, peak period and direction) obtained from the WAVEWATCH III model (NCEP NOAA) was statistically analyzed, in order to determine the central tendency and information variability. The program $R$ to tabulate and evaluate the information, do statistical calculations and graphical display. The annual time series analysis indicated the predominance of waves coming from East and South-East, with heights ranging from $0.39 \mathrm{~m}$ to $4.89 \mathrm{~m}$. The mean period indicated $a$ predominance of local wind-generated waves, with less than 10 seconds. The influence of waves generated in other regions of the ocean is evidenced by considering the standard deviation of the registry. The parameters tabulated monthly indicated that June, July, August and September are the most energetic months, which correspond to winter and spring seasons.
\end{abstract}




\section{INTRODUÇÃO}

O ambiente costeiro é altamente dinâmico e responde às condições forçantes como ondas, correntes e marés, apresentando um comportamento complexo. Essa complexa interação entre as condições forçantes e a morfologia costeira é característica de um ambiente morfodinâmico. O principal processo que causa mudanças morfológicas é o transporte de sedimentos.

Transporte de sedimentos sobre a plataforma continental é globalmente dominada por ondas de tempestade, correntes de maré e interação entre ondas e correntes oceânicas, entretanto a ressuspensão de sedimentos por ondas energéticas é o mecanismo dominante para o transporte de sedimentos na maioria das plataformas continentais do mundo (HARRIS \& WIBERG, 2001).

Nas zonas costeiras, o litoral, responde com mudanças de forma e de posição em função da atuação de parâmetros oceanográficos (ondas, marés e correntes costeiras), meteorológicos (precipitação e ventos) e hidrológicos (vazão fluvial) (MUEHE, 1995). Ocorrendo predominância de um parâmetro sobre outro, o ambiente costeiro se desenvolve até atingir seu equilíbrio dinâmico.

Estudos sobre as características das ondas atuantes na linha de costa são fundamentais para o entendimento do comportamento dinâmico das regiões costeiras. Obras de engenharia costeira, que incluem a instalação de estruturas portuárias, pontes, passarelas, avenidas à beira-mar prescindem de um conhecimento detalhado do comportamento das ondas no litoral.

Apesar da sua importância, a medição de parâmetros de ondas é insuficiente, pontual, curta ou inexistente para a caracterização do comportamento das ondas no litoral brasileiro, o que demanda a utilização de modelos matemáticos de geração e propagação de ondas através do uso de dados de ventos.

Séries temporais são conjuntos de observações da variabilidade de um parâmetro ao longo do tempo (ESQUIVEL, 2012); assim, longas séries temporais permitem estudos mais aprofundados do comportamento plurianual de determinado parâmetro.

A análise estatística de série temporal é uma seção da Ciência Estatística que se dedica a análise de eventos ou observações dependentes, permitindo a análise da frequência de ocorrência de determinado fenômeno além do seu padrão de comportamento imediato.

Os principais parâmetros que caracterizam uma onda são altura, comprimento, período e direção de aproximação. Estes são definidos pelas características do vento, tais como, intensidade, duração e área livre do oceano onde o vento atua (tamanho da pista) e pela distância da região de formação da agitação, diferindo ondas geradas pelo vento local (sea) de ondas provenientes de outras regiões (swell).

No Oceano Atlântico Sul, as ondas são geradas predominantemente em duas regiões: o cinturão de ventos alísios e o cinturão de tempestades de altas latitudes (DAVIES, 1972 apud DOMINGUEZ et. al., 1992). Na plataforma continental leste brasileira, o regime de ondas reflete o padrão de ventos locais e é caracterizado principalmente por ondas formadas pelos alísios de NE, mas este padrão pode ser modificado pelas ondas geradas por frentes frias polares e por eventos El Niño extremos (BITTENCOURT et. al., 2000).

\section{ÁREA DE ESTUDOS}

O município de Ilhéus está localizado no Litoral Sul do Estado da Bahia (Figura 1), possui uma área de unidade territorial de 1.584,69 $\mathrm{km}^{2}$ (IBGE, 2017), faz parte da zona turística Costa do Cacau e está inserido no bioma Mata Atlântica, apresentando clima classificado como tropical quente e úmido.

Ilhéus é uma região de micro/meso marés, apresentando nível médio da variação da maré mensal, nos últimos 11 anos (2005 - 2016) de 1.12 metros, e altura máxima prevista no ano de 2016 de 2.30 metros (CHM - Centro de Hidrografia da Marinha/ DHN, 2016).

A descarga fluvial na região é representada pelos rios Almada e Cachoeira que apesar de importantes para o abastecimento urbano e como ambientes de lazer, suas descargas médias anuais não são expressivas, sendo de $13.9 \mathrm{~m}^{3} / \mathrm{s}$ (Almada) e $24.06 \mathrm{~m}^{3} / \mathrm{s}$ (Cachoeira) (BAHIA, 2001).

Assim, no litoral de Ilhéus, as ondas representam a forçante hidrodinâmica mais importante na morfodinâmica costeira. 


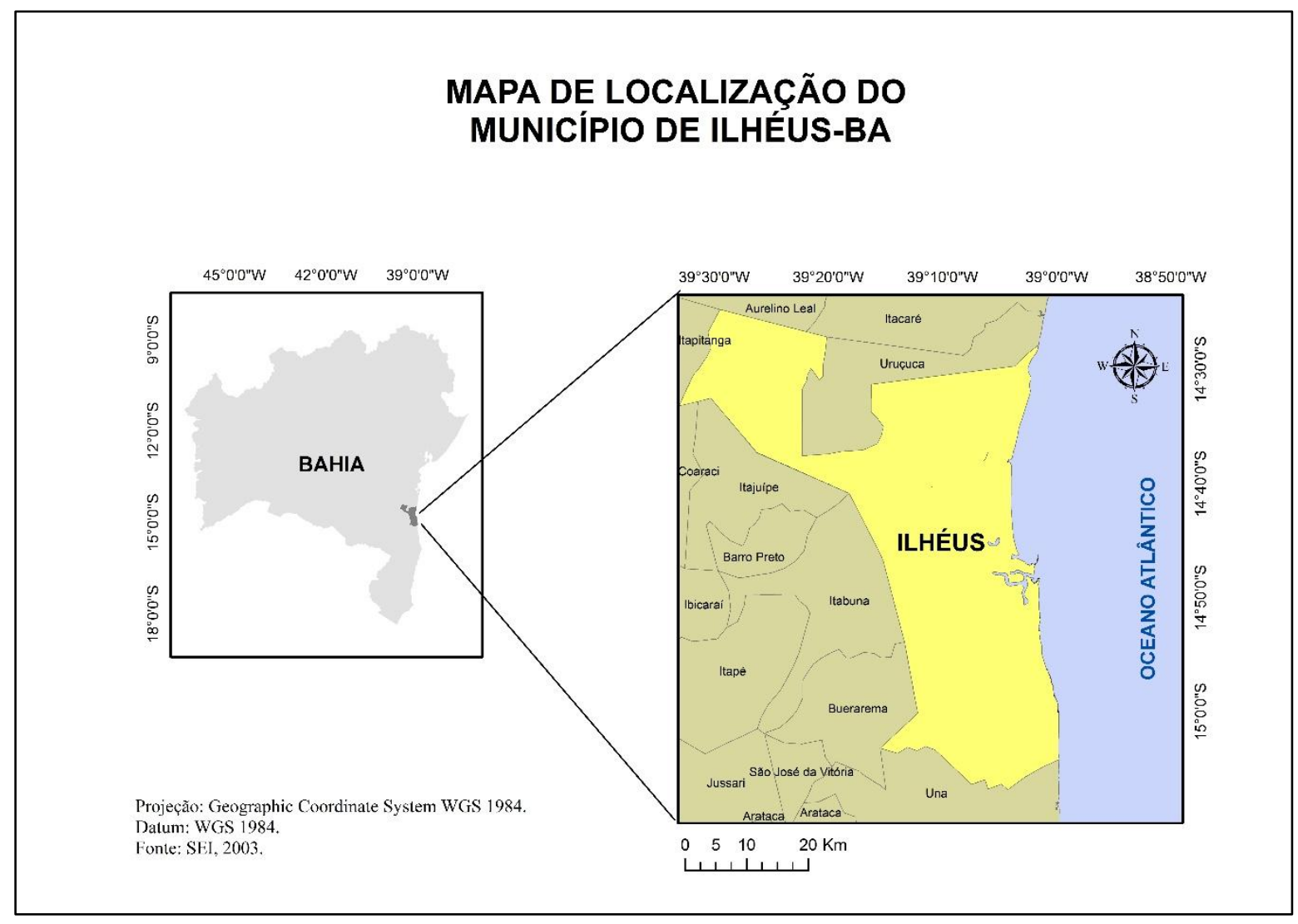

Figura 1: Mapa de Localização do município de Ilhéus-Ba, litoral sul da Bahia.

\section{MATERIAIS E MÉTODOS}

Recentemente, modelos matemáticos de geração e propagação de ondas através da utilização de dados de ventos têm sido muito utilizados em estudos de dinâmica costeira e de plataforma. Dentre eles podemos citar o modelo WAVEWATCH (TOLMAN, 1999) que resolve a equação do balanço de densidade espectral (espectro direcional da onda) e inclui crescimento, decaimento, interações não lineares, dissipação, fricção com o fundo, arrebentação e espalhamento da onda, a partir da reanálise de dados de vento do National Center for Environment Prediction (NCEP) do National Oceanic and Atmospheric Administration (NOAA) - United States.

As informações de saída do modelo, utilizadas nesta pesquisa (altura significativa, período de pico e direção média da onda), foram obtidas através do Centro de Modelagem Ambiental (ENVIRONMENTAL MODELING CENTER, 2016) um dos links disponíveis no site da NCEP/NOAA, ambiente virtual onde é possível ter acesso a descrição do modelo utilizado, bem como baixar os parâmetros da onda do banco de informações do modelo.

O modelo global do WAVEWATCH III fornece uma série temporal com informações a cada três horas $(00,03,06,09,12,15,18,21 \mathrm{~h})$, perfazendo um total de 8 informações diárias. Segundo Nascimento (2013), "a física do modelo inclui geração de energia, dissipação devido à quebra de ondas e fricção com o fundo, refração, advecção e interações onda-onda quadruplas". O modelo global desenvolvido pela MMAB (Marine Modeling and Analysis Branch) do NCEP tem a resolução espacial de $0.5^{\circ}$ longitude x $0.5^{\circ}$ latitude. Com sua última atualização, o WAVEWACTH III versão 3.14 passa a ser adequado para águas mais próximas das regiões costeiras (TOLMAN, 2009), gerando os parâmetros espectrais da onda usados neste trabalho: altura significativa (Hs), período de pico da onda $(\mathrm{Tp})$ e a direção média para o período de pico $(\mathrm{Dp})$, até uma profundidade mínima de 25 metros (a versão 2.22 tinha seu uso indicado apenas para águas mais profundas que $40 \mathrm{~m}$ ).

Foi utilizada uma estação para a aquisição dos parâmetros espectrais da onda simulada, afastada do continente para minimizar a influência das irregularidades da batimetria da plataforma continental da região. A série temporal foi obtida em frente a Ilhéus, nas coordenadas $15^{\circ}$ de latitude $\mathrm{S}$ e $37.5^{\circ}$ de longitude $\mathrm{W}$, num ponto com profundidade em torno dos 4000 metros. (Figura 2). 


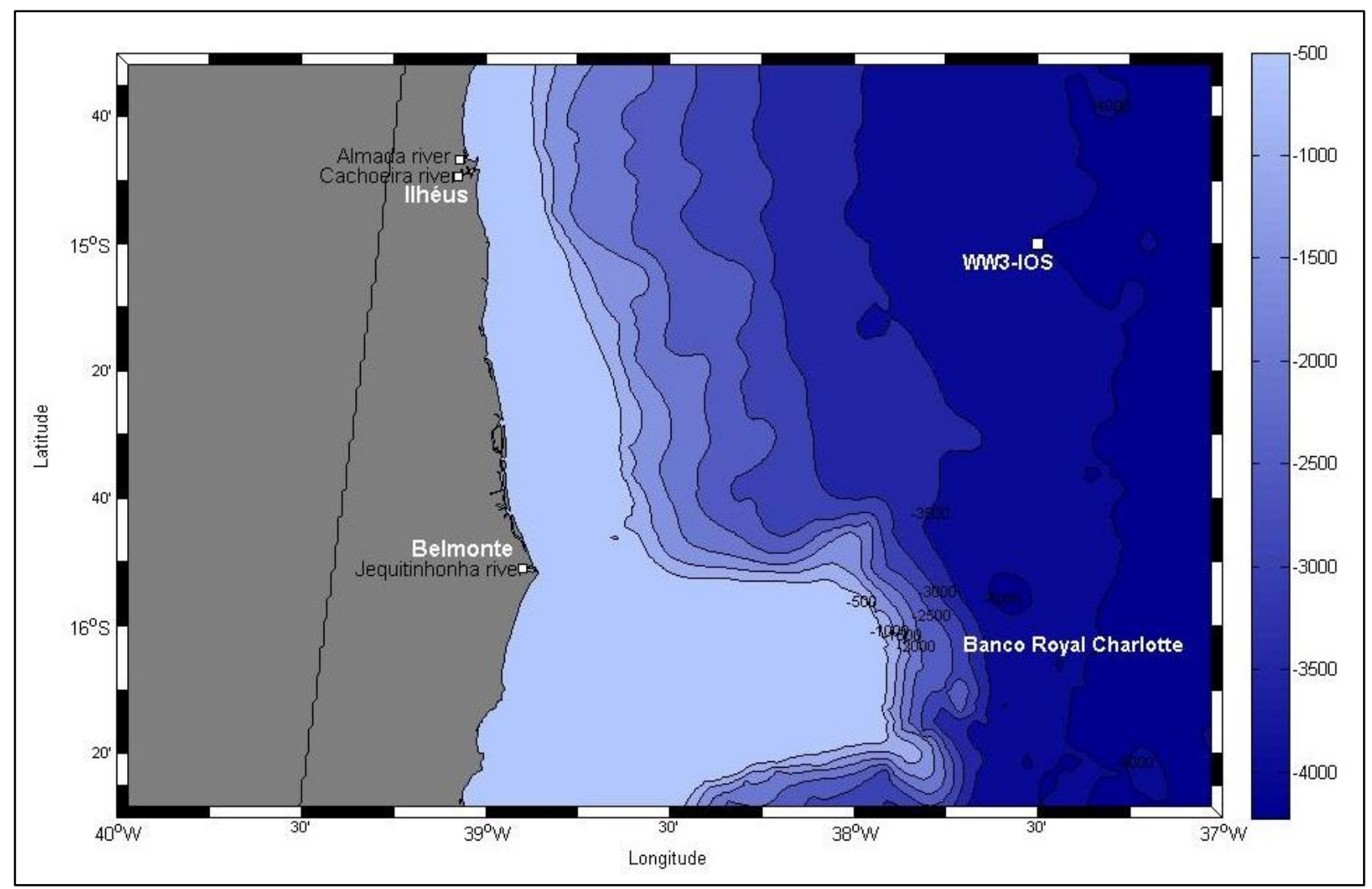

Figura 2: Mapa batimétrico da plataforma continental e ponto de aquisição das informações do WAVEWATCH III.

Para o arquivamento, organização e manipulação estatística de uma quantidade tão expressiva de dados (8 informações diárias x 365 ou 366 dias x 31 anos) foi utilizado o programa R. O programa R é um software livre criado por Ihaka e Gentleman (1996) que permite a manipulação, avaliação e interpretação estatística de dados, cálculo e exibição gráfica. A interface utilizada foi o Tinn-R (FARIA et al., 2010, 2014).

\section{RESULTADOS E DISCUSSÕES}

A análise estatística do banco de informações dos parâmetros de onda, obtidos do modelo WAVEWATCH III permitiu a determinação da altura média das ondas para os 31 anos da série temporal em $1.79 \mathrm{~m}$, com período $8.89 \mathrm{~s}$ e direção média de $116.95^{\circ}$ (quadrante Sudeste). Para melhor detalhamento da análise da série temporal, os parâmetros estatísticos foram analisados a cada ano (Tabela 1).

Na Tabela 1, podemos destacar na coluna de período médio, a ocorrência de valores inferiores a 10 segundos, demonstrando a predominância de ondas geradas pelo vento local (vagas / sea) no clima de ondas. No entanto, ao considerarmos o desvio padrão, que representa a medida de dispersão dos dados em torno da média, identificamos a influência das ondas geradas em outras regiões do oceano, pois observamos valores de período acima de 10 segundos, que indicam a influência de marulhos (swell). Os valores de desvio padrão mais elevados, como no ano de 2006 para altura da onda, indica maior dispersão do dado, ou seja, uma maior variabilidade no registro. O contrário é observado em 1987, indicando valores mais homogêneos. Quanto ao período, o registro mais homogêneo é observado nos anos de 2000 e 2005, enquanto a maior variabilidade ocorreu no ano de 1986. 
Tabela 1: Tabulação das médias, desvio padrão (SD) e variância (Var), para as informações de Hs (altura significativa), Tp (período de pico) e Dir (direção média) para cada ano da série temporal.

\begin{tabular}{|l|c|c|c|c|c|c|c|c|}
\hline Ano & $\begin{array}{c}\text { Hs } \\
\text { Média }\end{array}$ & SD - Hs & $\begin{array}{c}\text { Var - } \\
\text { Hs }\end{array}$ & $\begin{array}{c}\text { Tp } \\
\text { Média }\end{array}$ & SD - Tp & $\begin{array}{c}\text { Var - } \\
\text { Hs }\end{array}$ & $\begin{array}{c}\text { Dir } \\
\text { Média }\end{array}$ & $\begin{array}{c}\text { SD - } \\
\text { Dir }\end{array}$ \\
\hline $\mathbf{1 9 7 9}$ & 1.80 & 0.42 & 0.17 & 9.03 & 2.17 & 4.72 & 120.02 & 34.48 \\
\hline $\mathbf{1 9 8 0}$ & 1.76 & 0.34 & 0.11 & 8.61 & 2.05 & 4.18 & 11.74 & 33.06 \\
\hline $\mathbf{1 9 8 1}$ & 1.85 & 0.41 & 0.17 & 9.05 & 2.28 & 5.22 & 115.86 & 34.86 \\
\hline $\mathbf{1 9 8 2}$ & 1.85 & 0.39 & 0.15 & 9.03 & 2.45 & 5.99 & 116.78 & 34.41 \\
\hline $\mathbf{1 9 8 3}$ & 1.75 & 0.44 & 0.19 & 9.08 & 2.51 & 6.32 & 117.87 & 35.23 \\
\hline $\mathbf{1 9 8 4}$ & 1.78 & 0.46 & 0.21 & 9.58 & 2.47 & 6.09 & 126.06 & 37.46 \\
\hline $\mathbf{1 9 8 5}$ & 1.84 & 0.51 & 0.26 & 9.13 & 2.37 & 5.64 & 119.67 & 35.98 \\
\hline $\mathbf{1 9 8 6}$ & 1.78 & 0.45 & 0.20 & 9.27 & $\mathbf{2 . 5 5}$ & 6.53 & 121.92 & 36.48 \\
\hline $\mathbf{1 9 8 7}$ & 1.68 & $\mathbf{0 . 3 3}$ & 0.11 & 8.95 & 2.54 & 6.43 & 117.98 & 37.10 \\
\hline $\mathbf{1 9 8 8}$ & 1.83 & 0.55 & 0.30 & 9.03 & 2.32 & 5.38 & 118.92 & 33.16 \\
\hline $\mathbf{1 9 8 9}$ & 1.83 & 0.43 & 0.18 & 8.82 & 2.13 & 4.53 & 116.58 & 35.25 \\
\hline $\mathbf{1 9 9 0}$ & 1.75 & 0.42 & 0.18 & 9.22 & 2.44 & 5.94 & 120.19 & 35.03 \\
\hline $\mathbf{1 9 9 1}$ & 1.86 & 0.53 & 0.28 & 8.72 & 2.21 & 4.86 & 110.42 & 35.28 \\
\hline $\mathbf{1 9 9 2}$ & 1.83 & 0.40 & 0.16 & 8.77 & 2.03 & 4.12 & 109.64 & 30.82 \\
\hline $\mathbf{1 9 9 3}$ & 1.73 & 0.40 & 0.16 & 9.15 & 2.50 & 6.26 & 120.73 & 36.60 \\
\hline $\mathbf{1 9 9 4}$ & 1.77 & 0.38 & 0.14 & 8.81 & 2.17 & 4.71 & 116.77 & 32.76 \\
\hline $\mathbf{1 9 9 5}$ & 1.75 & 0.42 & 0.18 & 8.69 & 2.36 & 5.57 & 112.56 & 37.39 \\
\hline $\mathbf{1 9 9 6}$ & 1.76 & 0.50 & 0.25 & 8.73 & 2.35 & 5.54 & 119.87 & 34.20 \\
\hline $\mathbf{1 9 9 7}$ & 1.73 & 0.42 & 0.17 & 8.98 & 2.46 & 6.05 & 117.28 & 37.81 \\
\hline $\mathbf{1 9 9 8}$ & 1.83 & 0.44 & 0.19 & 8.83 & 2.18 & 4.77 & 118.60 & 34.09 \\
\hline $\mathbf{1 9 9 9}$ & 1.83 & 0.49 & 0.24 & 8.68 & 2.25 & 5.06 & 118.08 & 35.65 \\
\hline $\mathbf{2 0 0 0}$ & 1.80 & 0.42 & 0.18 & 8.49 & $\mathbf{1 . 8 1}$ & 3.29 & 112.42 & 33.05 \\
\hline $\mathbf{2 0 0 1}$ & 1.79 & 0.46 & 0.21 & 8.65 & 2.16 & 4.68 & 114.08 & 32.27 \\
\hline $\mathbf{2 0 0 2}$ & 1.77 & 0.41 & 0.17 & 8.55 & 2.12 & 4.49 & 111.96 & 35.84 \\
\hline $\mathbf{2 0 0 3}$ & 1.70 & 0.44 & 0.19 & 8.67 & 2.36 & 5.57 & 119.67 & 36.51 \\
\hline $\mathbf{2 0 0 4}$ & 1.80 & 0.38 & 0.15 & 8.82 & 2.20 & 4.82 & 118.27 & 34.78 \\
\hline $\mathbf{2 0 0 5}$ & 1.80 & 0.45 & 0.20 & 8.58 & $\mathbf{1 . 8 1}$ & 3.29 & 113.39 & 30.90 \\
\hline $\mathbf{2 0 0 6}$ & 1.87 & $\mathbf{0 . 5 6}$ & 0.32 & 8.89 & 2.18 & 4.76 & 118.53 & 33.94 \\
\hline $\mathbf{2 0 0 7}$ & 1.89 & 0.51 & 0.26 & 8.87 & 2.26 & 5.09 & 112.01 & 33.02 \\
\hline $\mathbf{2 0 0 8}$ & 1.81 & 0.46 & 0.21 & 9.17 & 2.19 & 4.78 & 122.36 & 35.72 \\
\hline $\mathbf{2 0 0 9}$ & 1.76 & 0.45 & 0.20 & 8.97 & 2.45 & 5.98 & 115.24 & 37.11 \\
\hline & & & & & & & & \\
\hline
\end{tabular}

Objetivando analisar a variabilidade mensal dos parâmetros das ondas, foram determinados os parâmetros estatísticos das ondas para cada mês de toda a série temporal (Tabela 2).

As variações na altura da onda, que constam na Tabela 2, apresentaram mínimo de $0.39 \mathrm{~m}$ (janeiro) com seu máximo de $4.89 \mathrm{~m}$ (setembro). Ao observamos as colunas de Hs min e Hs max, constatamos que os meses de junho, julho, agosto e setembro apresentam valores de altura mínima das ondas superiores a 1 metro e com as alturas máximas superiores a 4 metros; considerando que a energia da onda é diretamente proporcional ao quadrado de sua altura (KINSMAN, 1984), estes são os meses mais energéticos.

Podemos destacar uma diferença de padrão no mês de abril, onde o máximo foi de 4.30 metros no ano de 1981, com a altura mínima de 1.24 metros, períodos variando de 7.36 a 15.26 segundos e as direções variando de $92.42^{\circ}$ (quadrante leste) a $193.05^{\circ}$ (quadrante sul). Ao conferirmos a tabela de ONI (Oceanic Niño Index) que monitora a oscilação da temperatura das águas superficiais do Oceano Pacífico em relação a fenômenos oceânicos e atmosféricos, por conta dos eventos El Niño e La Niña no hemisfério sul (CLIMATE PREDICTION CENTER, 2017), constatamos que em 1981, nos meses de março, abril e maio houve 
variação negativa da temperatura em apenas 0.4 graus, não sendo possível associar com o fenômeno climático La Niña, pois a ocorrência de tais eventos só é registrada quando as variações de temperatura superam $0.5^{\circ} \mathrm{C}$ positivo ou negativo. Estudos mais aprofundados poderão identificar outros parâmetros que contribuam para esclarecer esta alteração observada no mês de abril de 1981, em comparação com os demais anos.

Tabela 2: Análise estatística dos parâmetros de ondas, da série de 31 anos, valor do total anual e mensal da série. Altura significativa (Hs) em metros, Período de pico (Tp) em segundos e Direção (Dir) em graus.

\begin{tabular}{|l|l|l|l|l|l|l|l|l|l|l|l|l|}
\hline Meses & $\begin{array}{l}\text { Hs } \\
\text { min }\end{array}$ & $\begin{array}{l}\text { Hs } \\
\text { max }\end{array}$ & $\begin{array}{l}\text { Hs } \\
\text { range }\end{array}$ & $\begin{array}{l}\text { Hs } \\
\text { média }\end{array}$ & $\begin{array}{l}\text { Hs } \\
\text { SD }\end{array}$ & $\begin{array}{l}\text { Tp } \\
\text { min }\end{array}$ & $\begin{array}{l}\text { Tp } \\
\text { max }\end{array}$ & $\begin{array}{l}\text { Tp } \\
\text { range }\end{array}$ & $\begin{array}{l}\text { Tp } \\
\text { média }\end{array}$ & $\begin{array}{l}\text { Tp } \\
\text { SD }\end{array}$ & $\begin{array}{l}\text { Dir } \\
\text { média }\end{array}$ & $\begin{array}{l}\text { Dir } \\
\text { SD }\end{array}$ \\
\hline 31 anos & 0.39 & 4.89 & 4.50 & 1.79 & 0.45 & 2.37 & 20.11 & 17.74 & 8.90 & 2.29 & 116.97 & 35.10 \\
\hline janeiro & 0.39 & 3.11 & 2.72 & 1.54 & 0.27 & 2.37 & 17.70 & 15.33 & 7.76 & 1.89 & 100.46 & 32.70 \\
\hline fevereiro & 0.75 & 2.90 & 2.15 & 1.50 & 0.29 & 4.59 & 18.20 & 13.61 & 8.22 & 2.30 & 110.20 & 34.85 \\
\hline março & 0.80 & 3.12 & 2.32 & 1.54 & 0.30 & 4.31 & 20.11 & 15.80 & 8.99 & 2.54 & 121.16 & 36.26 \\
\hline abril & 0.86 & 4.30 & 3.44 & 1.69 & 0.37 & 4.69 & 19.40 & 14.71 & 9.35 & 2.41 & 126.98 & 34.50 \\
\hline maio & 0.86 & 3.89 & 3.03 & 1.87 & 0.45 & 5.28 & 19.22 & 13.94 & 9.85 & 2.56 & 132.75 & 35.58 \\
\hline junho & 1.03 & 4.20 & 3.17 & 2.02 & 0.48 & 4.49 & 18.11 & 13.62 & 9.44 & 2.35 & 121.56 & 33.41 \\
\hline julho & 1.03 & 4.54 & 3.51 & 2.12 & 0.51 & 4.76 & 19.14 & 14.38 & 9.27 & 1.94 & 118.30 & 31.52 \\
\hline agosto & 1.03 & 4.25 & 3.22 & 2.08 & 0.50 & 4.68 & 19.33 & 14.65 & 9.31 & 2.06 & 117.69 & 30.47 \\
\hline setembro & 1.01 & 4.89 & 3.88 & 1.97 & 0.48 & 4.94 & 18.41 & 13.47 & 9.53 & 2.16 & 126.62 & 33.21 \\
\hline outubro & 1.03 & 3.69 & 2.66 & 1.79 & 0.34 & 4.68 & 17.74 & 13.06 & 8.76 & 2.06 & 115.88 & 33.05 \\
\hline novembro & 0.92 & 3.54 & 2.62 & 1.76 & 0.33 & 4.80 & 19.36 & 14.56 & 8.40 & 2.04 & 109.77 & 34.54 \\
\hline dezembro & 0.79 & 3.76 & 2.97 & 1.59 & 0.29 & 4.29 & 16.73 & 12.44 & 7.87 & 1.85 & 101.99 & 34.92 \\
\hline
\end{tabular}

\section{CONCLUSÕES}

O comportamento das ondas atuantes na faixa oceânica do município de Ilhéus pode ser caracterizado pela predominância de ondas do quadrante SE (direção média $116.97^{\circ}$ ), com altura média de 1.79 m e período médio de 8.90 s para o intervalo de 1979 a 2009. A análise mensal dos parâmetros da onda indicou a predominância de ondas do quadrante Leste $\left(67.5\right.$ a $\left.112.5^{\circ}\right)$, nos meses de novembro $\left(109.77^{\circ}\right)$, dezembro $\left(101.99^{\circ}\right)$, janeiro $\left(100.46^{\circ}\right)$ e fevereiro $\left(110.20^{\circ}\right)$, que representam o final da estação de primavera (novembro) e todo o período de verão (dezembro, janeiro e fevereiro), de acordo com a direção média. Tais características correspondem à constância de direção e velocidade dos ventos alísios (Zona de Convergência Intertropical), que nas estações de verão e outono, posiciona-se mais a sul, sendo a principal forçante da geração de ondas do quadrante Leste nessa região (AYOADE, 2010; DOMINGUEZ et al., 1992; REBOITA et al., 2012).

O sistema atmosférico mais importante para o litoral do Nordeste é a Zona de Convergência Intertropical (ZCIT). Nas estações de verão e outono, este sistema atmosférico, posiciona-se mais a sul, já nas estações de inverno e primavera a ZCIT migra para o norte (REBOITA et al., 2012), nesse período a associação com outros sistemas meteorológicos, tais como a Zona de Convergência do Atlântico Sul (ZCAS), Linhas de Instabilidade (Lis) e Distúrbio Ondulatórios de Leste (DOLs) são de extrema importância para a definição da qualidade do período chuvoso das Regiões Norte e Nordeste do Brasil, assim como os padrões de vento (MELO et al., 2009), que interferem diretamente na caracterização do comportamento das ondas em termos de altura, período e direção, podendo ser identificada uma maior ocorrência de ondas provenientes de outras regiões do oceano, denominadas de marulhos (swell), caracterizadas por apresentarem maiores alturas e períodos, sendo os períodos de maior poder das ondas na costa.

Em Ilhéus, os meses mais energéticos são junho, julho, agosto (inverno) e setembro (primavera), com mínimas acima de 1 metro e máximas acima dos 4 metros. A altura mais baixa da série temporal de 31 anos do modelo WAVEWATCH III foi registrada em janeiro $(0.39 \mathrm{~m})$ e a mais alta em setembro $(4.89 \mathrm{~m})$

A análise estatística da série temporal de 31 anos de informações de altura significativa, período de pico e direção média das ondas do modelo WAVEWATCH III, apresentada neste trabalho, disponibiliza informações que poderão ser utilizadas como condições de contorno em modelos de propagação de ondas 
sobre a plataforma continental e em estudos de dinâmica costeira preenchendo uma lacuna no conhecimento sobre o clima de ondas na costa brasileira.

\section{BIBLIOGRAFIA}

AYOADE, J.O. Introdução à Climatologia para os Trópicos.13. Rio de Janeiro. Ed. Bertrand Brasil, (tradutor; Maria Juracy Zani dos Santos). 332p. 2010.

BAHIA. Convênio SRH - UESC. Programa de Recuperação das Bacias dos Rios Cachoeira e Almada: Diagnóstico Regional - Caracterização Hidrológica. Vol. I, Tomo IV. 46p. 2001.

BITTENCOURT, A. C. S. P.; DOMINGUEZ, J. M.; MARTIN, L.; SILVA, I. R. Patterns of Sediment Dispersion Coastwise the State of Bahia - Brazil. Anais da Academia Brasileira de Ciências, v. 72, n. 2, pp. 271-287. 2000. Disponível em: $<$ http://www.scielo.br/pdf/aabc/v72n2/0026.pdf $>$ Acesso em: jun/2016.

CENTRO DE HIDROGRAFIA DA MARINHA (Marinha do Brasil), Tabua das Marés do Brasil. Rio de Janeiro, 2017. Disponível em: <http://www.mar.mil.br/dhn/chm/box-previsaomare/tabuas/40145Jan2017.htm>. Acesso em: 05 jan. 2017.

CLIMATE PREDICTION CENTER. Oceanic Niño Index (ONI) Disponível em: http://www.cpc.ncep.noaa.gov/products/analysis monitoring/ensostuff/ensoyears.shtml Acesso em: $\mathrm{jan} / 2017$

DOMINGUEZ, J.M.L.; BITTENCOURT, A.C.S.P. and MARTIN, L. Controls on Quaternary coastal evolution of the east-north-eastern coast of Brazil: roles of sea-level history, trade winds and climate. In: J.F. Donoghue, R.A. Davis, C.H. Fletcher and J.R. Suter (Editors), Quaternary Coastal Evolution. Sediment. Geol., 80: 213-232. 1992.

ENVIRONMENTAL MODELING CENTER. DOWNLOAD WAVEWATCH III. 2016. Disponível em: <ftp://polar.ncep.noaa.gov/pub/history/waves〉. Acesso em: fev 2016.

ESQUIVEL, R. de M. Análise Espectral Singular: Modelagem de séries temporais através de estudos comparativos usando diferentes estratégia de previsão. 2012. p 160. Dissertação (Mestrado em Modelagem Computacional e Tecnologia Industrial). SENAI CIMATEC. Salvador, 2012. Disponível em: <http://portais.fieb.org.br/portal faculdades/images/portal/NRM/DissertacoesMCTI/renataesquiveldisserta cao15junh12.pdf>. Acesso em: 08 jun. 2016.

FARIA, J. C.; GROSJEAN, P.; JELIHOVSCHI, E.; PIETROBON, R. Tinn-R Editor. Zurich: RMETRICS ASSOCIATION, 2010. 199p.

FARIA, J. C.; GROSJEAN, P.; JELIHOVSCHI, E.; PIETROBON, R. Tinn-R Editor - GUI for R language and Environment. 2. ed. Ilhéus: Editus - Editora da UESC, v. 1. 267p. 2014.

HARRIS, C.K., WIBERG, P.L. A two-dimensional, time-dependent model of suspended sediment transport and bed reworking for Continental Shelves. Computers \& Geosciences 27: 675-690. 2001.

IBGE, Instituto Brasileiro de Geografia e Estatistica. 2017. Disponível em: <http://cidades.ibge.gov.br/xtras/perfil.php?lang=\&codmun=291360\&search=bahia|ilheus $>$. Acesso em :08 jun. 2017.

IHAKA, R., GENTLEMAN, R. R: A language for data analysis and graphics. Journal of Computation and Graphical Statistics. V.5, n3. 299-314, 1996.

KINSMAN, B., Wind Waves: Their Generation and Propagation on the Ocean Surface. New York: Dover Publications, Inc. 700p. 1984.

MELO, A. B. C. de; CAVALCANTI, I. F. de A.; SOUZA, P. P., Zona de Convergência Intertropical do Atlântico. In: CAVALCANTI, I. F. de A.; FERREIRA, N. J.; SILVA M. G. A. J. da.; DIAS, M. A. F. da. S. (org.). Tempo e Clima: no Brasil. São Paulo, SP, Ed. Oficina de Textos. cap.2, p. 25-39. 2009.

MUEHE, D., Geomorfologia costeira. In: GUERRA, A. J. T. \& CUNHA, S. B. (org.). Geomorfologia: uma atualização de bases e conceitos. Rio de Janeiro, RJ, Ed. Bertrand Brasil. cap. 6, p. 253-308. 1995.

REBOITA, M.S.; KRUSCHE, N.; AMBRIZZI, T.; ROCHA, R. P. da. Entendendo o Tempo e o Clima na América do Sul. Terra Didática, Campinas - SP, 8, 1, 34-50, 2012. Disponível em: 〈https://www.ige.unicamp.br/terraedidatica/v8-1/pdf81/s3.pdf >. Acesso em: 08 abril 2017. 
TOLMAN, H. L. User manual and system documentation of WAVEWATCH III version 1.18.NOAA/NWS/NCEP/OMB. Tech. Note 166, 1999. 110 p. Disponível em: < http://polar.ncep.noaa.gov/mmab/papers/tn166/OMB 166.pdf>. Acesso em: 07 jan. 2016.

TOLMAN, H. L. User manual and system documentation of WAVEWATCH III version 3.14. NOAA / NWS / NCEP / MMAB Technical Note 276, 2009.194 pp.+ Appendices. Disponível em: <http://polar.ncep.noaa.gov/mmab/papers/tn276/MMAB_276.pdf >. Acesso em: 07 jan. 2016. 